Z Gerontol Geriat 2016 • 49:179-180 DOI 10.1007/s00391-016-1048-7

Online publiziert: 4. April 2016

๑) Springer-Verlag Berlin Heidelberg 2016

CrossMark
Cornelia Kricheldorff' ' Walter Hewer ${ }^{2}$

'Katholische Hochschule Freiburg, Freiburg, Deutschland

${ }^{2}$ Klinikum Christophsbad, Göppingen, Deutschland

\title{
Versorgung von Menschen mit Demenz im gesellschaftlichen Wandel
}

Der demografische Wandel, v. a. im Hinblick auf die zahlenmäßig deutliche Zunahme von hochbetagten Menschen, führt schon heute zu einer erhöhten Zahl von demenziellen Erkrankungen. Bei aktuell mindestens 1,2 Mio. Menschen mit Demenz kommen jährlich ca. 250.000 Neuerkrankungen hinzu. Auf Basis eines Datensatzes der AOK wird davon ausgegangen, dass im Jahr 2050 bis zu 3 Mio. Menschen von einer Demenzerkrankung betroffen sein werden [1]. Gleichzeitig vollziehen sich bedeutsame gesellschaftliche Wandlungsprozesse, die $\mathrm{zu}$ verstärkter Pluralisierung und steigender Singularisierungstendenz führen und die traditionellen Versorgungsformen für Menschen mit Demenz brüchig werden lassen. Diese sind v. a. in frühen und mittleren Stadien der Demenz in den familiären Netzwerken verortet, die aber, aufgrund häufig mangelnder Vereinbarkeit von Sorgeaufgaben und Beruf sowie verstärkter Mobilität, die mit der familiären Pflege verbundenen Aufgaben immer weniger wahrnehmen können.

Bedarfsgerechte Versorgungssettings für Menschen mit Demenz müssen sich an diesen gesellschaftlichen Entwicklungen orientieren und neue Wege erproben. Dabei geht es einerseits um den Versuch, die fehlenden Ressourcen im familiären Bereich sowie in Sozialraum und Quartier durch neu geschaffene Strukturen zumindest teilweise zu substituieren [2, 3]. Andererseits müssen sich Angebote der stationären Langzeitpflege mit neuen Ansätzen und Konzepten an den veränderten Erwartungen und $\mathrm{Be}$ dingungen orientieren. Mit den gesetz- lichen Neuregelungen des alten Heimrechts in immer mehr deutschen Bundesländern verbessern sich die Bedingungen für die Schaffung gemeinschaftlicher, kleiner Wohn- und Versorgungseinheiten, wie Wohngemeinschaften für Menschen mit Demenz, und alternative Pflegesettings, die ihre konzeptionellen Vorbilder vielfach in den west- und nordeuropäischen Nachbarländern haben. In vielen deutschen Kommunen entstehen derzeit Versorgungsnetzwerke, die unterschiedlich strukturiert und gesteuert werden, z. B. im Rahmen der bundesweiten Aktion „Lokale Allianzen für Menschen mit Demenz".

Aktuell ist also viel Bewegung in der Versorgungslandschaft, auf die auch die Förderpolitik entsprechend reagiert und damit Forschungsvorhaben und Modellprojekte möglich macht. Ein Beispiel dafür ist das Forschungsprogramm „Zukunftswerkstatt Demenz", vom Bundesgesundheitsministerium gefördert in den Jahren 2012-2015. In diesem Rahmen wurden 6 bereits bestehende und evaluierte Projekte zur Unterstützung pflegender Angehöriger von Menschen mit Demenz inhaltlich nochmals weiterentwickelt und evaluiert. In einer zweiten Förderlinie wurden regionale Demenznetzwerke in Bezug auf erkennbare Erfolgsfaktoren zur nachhaltigen Implementierung in der Praxis untersucht.

Im Rahmen unseres Themenschwerpunkts in der Zeitschrift für Gerontologie und Geriatrie werden, neben anderen Beiträgen, 3 dieser geförderten Projekte skizziert und ihre relevanten Forschungs- und Entwicklungsergebnisse vorgestellt. Den Auftakt macht der Beitrag von Monika Reichert, Sarah Hampel und Verena Reuter zum Projekt „Mobile Demenzberatung als niedrigschwelliges Hilfeangebot für pflegende Angehörige." Beschrieben werden die Chancen und Herausforderungen der Implementierung eines mobilen gerontopsychiatrischen Beratungsangebots, die in diesem Kontext erkennbaren Bedarfe der Nutzerinnen und Nutzer sowie die positiven Effekte der Beratung auf das Versorgungssetting der pflegenden Angehörigen. Dieses Angebot hat eine hohe Relevanz für Angehörige, die die Komm-Strukturen der einschlägigen Beratungsstellen nicht nutzen, aber in ihrer Alltagswelt, in ihrem Sozialraum erreicht und angesprochen werden können.

Mit dem Titel „EduKation demenz ${ }^{\circledR}$ psychoedukatives Schulungsprogramm für Angehörige von Menschen mit Demenz" stellen im anschließenden Beitrag die Autorinnen Sabine Engel und Andrea Reiter-Jäschke die hypothesengeleitete Überprüfung ihrer Intervention und die Ergebnisse der dazu durchgeführten statistischen Analysen vor. Die Untersuchungsergebnisse sind für die Praxis von großer Relevanz, weil sie nachweisen, dass durch spezifische Interventionen das psychosoziale Wohlbefinden von Angehörigen signifikant verbessert und deren Einstellung zu ihren demenzkranken Familienmitgliedern verändert werden kann. Positive Wirkungen gibt es in Bezug auf eine verständnisvollere und stärker akzeptierende Haltung, einen einfühlsameren kommunikativen Umgang und eine Verbesserung der Beziehungsqualität. 
Im dritten Beitrag von Ralf Ihl, $\mathrm{Na}$ dine Cujai und Katrin Krah beschreiben die Autor(inn)en die Arbeitsweise eines in einer Versorgungsregion implementierten kompletten Unterstützungsnetzwerks. Anhand einer Verlaufsuntersuchung bei einer Stichprobe älterer psychisch kranker Menschen - die Hälfte davon von einer Demenz betroffen - gehen sie der Frage nach, wie und in welchem Umfang der Umzug von der Häuslichkeit in die stationäre Pflege durch ein solches Netzwerk verhindert bzw. verzögert werden kann. Die Ergebnisse sprechen sehr deutlich für eine stabilisierende Funktion des Netzwerks in Bezug auf den Verbleib im häuslichen Umfeld. Dabei heben die Autor(inn)en hervor, dass solche Effekte durch Koordinierung vorhandener Ressourcen ohne zusätzliche Investitionen erreicht werden können.

Im Mittelpunkt des vierten Beitrags von Cornelia Kricheldorff und Thomas Brijoux stehen die zentralen Ergebnisse des Projekts „FABEL - Familienbegleitung bei Demenz im ländlichen Raum“, ebenfalls gefördert im Rahmen der „Zukunftswerkstatt Demenz“. Zentral sind dabei die Entwicklung und Erprobung des Curriculums zur Qualifizierung Freiwilliger, die die Familienbegleitung im häuslichen Bereich anbieten. Im Rahmen der begleitenden Forschung standen die möglichen Wirkungen dieser neuen Intervention, bei der die Belastung der pflegenden Angehörigen über den Studienzeitraum hinweg kontrolliert und mit einer Kontrollgruppe verglichen wurde.

Den Abschluss des Themenschwerpunkts bildet ein Beitrag von Judith Franzmann, Julia Haberstroh und Johannes Pantel zum Programm „Train the trainer in dementia care." Dabei geht es um die Verbesserung der demenzspezifischen sozial-kommunikativen Kompetenzen von Altenpflegekräften in der stationären Altenhilfe unter Anwendung des TANDEM-Programms, dessen Wirksamkeit bereits in früheren Studien nachgewiesen worden war. Neu an der hier publizierten Studie ist die Evaluation eines Trainings von aus der Altenhilfe kommenden Multiplikatoren. Es werden Daten einer Interventionsstudie vorgestellt, wonach über die Qualifizierung von Multiplikatoren positive Wirkungen in Bezug auf berufliche Belastungen und demenzspezifische soziale Kompetenzen von Pflegekräften erzielt werden können.

Die Botschaft unseres Schwerpunkthefts steht in vollem Einklang mit wesentlichen Empfehlungen der jüngst neu erschienenen S3-Leitlinie „Demenzen“ - AWMF-Register-Nr. 038/013 [4]. In dieser wird der Stellenwert einer breiten Palette psychosozialer Interventionen, reichend von kognitiver Stimulation bis hin zu künstlerischen Therapien, deutlich hervorgehoben. Explizit werden in diesem Kontext auch Maßnahmen genannt, die die Stärkung der Kompetenz der Angehörigen zum Ziel haben. Ebenso wird in der Leitlinie der Schutz der Gesundheit pflegender Angehöriger als ein originärer Bestandteil eines umfassenden Versorgungskonzepts für Demenzkranke definiert, und es werden evidenzbasierte Aussagen dazu getroffen. Insgesamt werden mit dem Themenschwerpunkt neue Optionen beleuchtet, die dazu beitragen, die Versorgungsqualität von Menschen mit Demenz zu verbessern und Versorgungssettings zu etablieren, die den sich verändernden gesellschaftlichen Rahmenbedingungen Rechnung tragen.

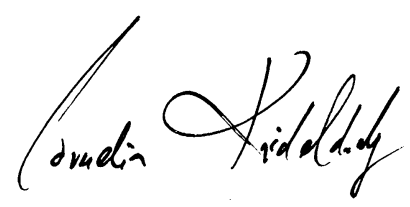

Prof. Dr. phil. Cornelia Kricheldorff

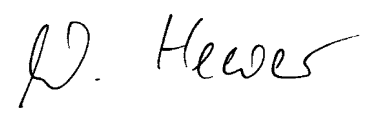

Prof. Dr. med. Walter Hewer

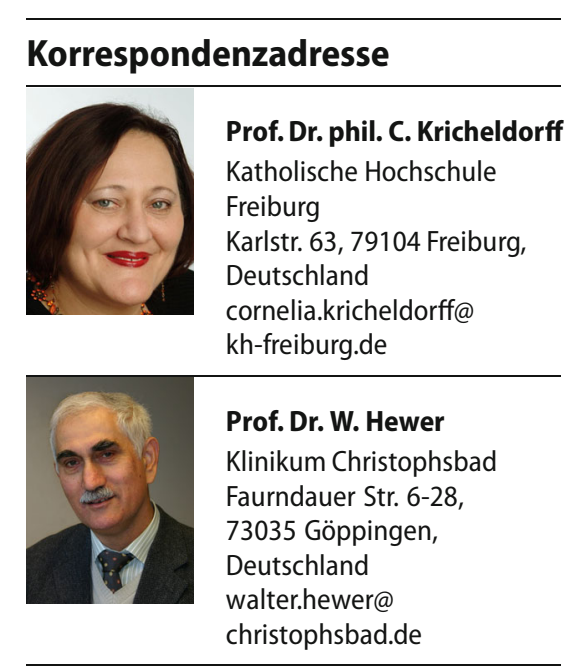

Interessenkonflikt. C. Kricheldorff und W. Hewer geben an, dass kein Interessenkonflikt besteht.

\section{Literatur}

1. Doblhammer G, Kreft D, Dethloff A (2012) Gewonnene Lebensjahre: langfristige Trends der Sterblichkeit nach Todesursachen in Deutschland und im internationalen Vergleich. Bundesgesundheitsblatt Gesundheitsforschung Gesundheitsschutz 55(4):448-458

2. KricheldorffC, BrijouxT(2015) Familienbegleitung Freiwilliges Engagement in der Begleitung von Familien bei Demenz. Manual - Handreichungen Materialien. Pabst Science, Lengerich

3. Bubolz-LutzE, KricheldorffC, GKV-Spitzenverband. (Hrsg) (2011) Pflegebegleiter. Schriftenreihe Modellprogramm zur Weiterentwicklung der Pflegeversicherung Bd.6.CW Haarfeld, Hürth

4. S3-Leitlinie „Demenzen“; AWMF Register Nummer: 038/013. Langversion - Januar 2016. https://www.dgppn.de/fileadmin/user_upload/_ medien/download/pdf/kurzversion-leitlinien/ S3-LL-Demenzen-240116-1.pdf. Zugegriffen: 8.2.2016 